\title{
Role of the Cerebellar Cortex in Conditioned Goal-Directed Behavior
}

\author{
Eric Burguière, ${ }^{1 \star}$ Arnaud Arabo, ${ }^{1 \star}$ Frederic Jarlier, ${ }^{1}$ Chris I. De Zeeuw, ${ }^{2,3}$ and Laure Rondi-Reig ${ }^{1}$ \\ ${ }^{1}$ Navigation, Memory and Aging Team, UMR CNRS 7102, Universite Pierre et Marie Curie, F-75005, Paris, France, ${ }^{2}$ Department of Neuroscience, Erasmus \\ Medical Center, 3000 DR, Rotterdam, The Netherlands, and ${ }^{3}$ Netherlands Institute for Neuroscience, Royal Dutch Academy of Arts and Sciences, \\ Meibergdreef 47, Amsterdam, The Netherlands
}

Learning a new goal-directed behavioral task often requires the improvement of at least two processes, including an enhanced stimulusresponse association and an optimization of the execution of the motor response. The cerebellum has recently been shown to play a role in acquiring goal-directed behavior, but it is unclear to what extent it contributes to a change in the stimulus-response association and/or the optimization of the execution of the motor response. We therefore designed the stimulus-dependent water Y-maze conditioning task, which allows discrimination between both processes, and we subsequently subjected Purkinje cell-specific mutant mice to this new task. The mouse mutants L7-PKCi, which suffer from impaired PKC-dependent processes such as parallel fiber to Purkinje cell long-term depression (PF-PC LTD), were able to acquire the stimulus-response association, but exhibited a reduced optimization of their motor performance. These data show that PF-PC LTD is not required for learning a stimulus-response association, but they do suggest that a PKC-dependent process in cerebellar Purkinje cells is required for optimization of motor responses.

\section{Introduction}

Adaptive processes are formed by adjustments of motor responses to varying environmental conditions. Associative learning includes both acquiring new associations between a given stimulus or context and a particular response, and adapting this response accurately to optimize behavior. These two components of associative learning are intrinsic components of classical or operant conditioning, wherein an animal learns to link a neutral stimulus [which becomes the conditioned stimulus (CS)] with a behavioral response [or conditioned response (CR)] to precisely prevent an aversive stimulus [or unconditioned stimulus (US)] (Fanselow and Poulos, 2005).

Investigations have been focused on the neural and cellular bases of this form of conditioning. Long-term synaptic depression (LTD) at the parallel fiber to Purkinje cell (PF-PC) synapses in the cerebellum has been proposed to be a major neural substrate for conditioning tasks (Ito and Kano, 1982). Interfering with specific cellular substrates, for example using protein kinase $\mathrm{C}$ inhibitors (PKCi), has been shown to block LTD induction at

\footnotetext{
Received April 26, 2010; revised May 24, 2010; accepted July 26, 2010.

This work was supported by grants from the ACI Neurosciences Intégratives et Computationnelles (NIC 0083), Fondation Recherche Médicale (programme Longévité Cognitive et Neurosensorielle-DLC20060206428), and Agence Nationale de la Recherche (ANR Young Researcher 07-JCJC-0108-01) (to L.R.R.); French Ministry of Research and Lilly Foundation fellowships (to E.B.); and Dutch Medical Research Council (ZON-MW), Dutch Scientific Council (NWO), European Union Research Program (SENSOPAC, C7, and CEREBNET), Prinses Beatrix Fonds, and Neuro-Bsik (to C.I.D.Z.). We thank Vanessa Gautheron, Boris Goncalves, and France Maloumian for technical support and Mark Howe for careful reading of the manuscript.

*E.B. and A.A. contributed equally to the work.

Correspondence should be addressed to either Laure Rondi-Reig at the above address or Eric Burguière at his present address: McGovern Institute for Brain Research, Massachusetts Institute of Technology, 46-6133, 43 Vassar Street, Cambridge, MA 02139. E-mail: laure.rondi@snv.jussieu.fr or eric_b@mit.edu.

DOI:10.1523/JNEUROSCI.2190-10.2010

Copyright $\odot 2010$ the authors $\quad 0270-6474 / 10 / 3013265-07 \$ 15.00 / 0$
}

the PF-PC neuron synapse in cultured Purkinje neurons (Linden and Connor, 1991). Later, several mice mutants with a blockade of LTD induction were generated by affecting PKC, mGluR1, PKG, and CaMKII pathways. With these mutants, authors have reported impairments either in the eyeblink conditioning task (Koekkoek et al., 2003) or in the adaptation of the vestibuloocular reflex (Aiba et al., 1994; De Zeeuw et al., 1998; Feil et al., 2003; Hansel et al., 2006). We also recently reported that elaboration of a direct path in a goal-directed navigation task is impaired in the L7-PKCi mouse mutant in which induction of cerebellar PF-PC LTD is blocked by inhibiting PKC (Burguière et al., 2005). Together, these studies pointed to a possible role of PF-PC LTD in the stimulus-response (S-R) association process and in temporal adjustment of the response. However, the role of the PF-PC LTD in such conditioning or goal-directed tasks is still debated, since these studies did not allow to dissociate impairments in the S-R association from deficits in execution of the motor response (Thompson and Steinmetz, 2009). In fact, in a recent pharmacological study where PF-PC LTD was selectively prevented in vivo, authors could not find any deficit in either motor learning or conditioning tasks (Welsh et al., 2005). In another study using a Lurcher mouse model with extensive cerebellar degeneration, the authors were still able to observed preserved conditioning with a selective impairment on performance (Porras-Garcia et al., 2005).

To determine the role of the Purkinje cells PKC-dependent processes, and particularly the PF-PC LTD, we compared L7PKCi mutant mice and their control littermates in a new stimulus-dependent water Y-maze (SWYM) conditioning task that requires a goal-directed behavior. In this SWYM task, mice had to learn to associate the correct turn (i.e., the CR) leading to the goal with the CS presented before the turn. We report here 
two main results from these experiments. First, that PF-PC LTD is not required for the acquisition of $\mathrm{S}-\mathrm{R}$ association in the SWYM task, but second, PF-PC LTD and/or other PKC-dependent processes are required to optimize the conditioned motor response resulting from this association.

\section{Materials and Methods}

Experimental subjects. We used male heterozygous transgenic L7-PKCi (-/L7-PKCi) mice and their wild-type $(-/-)$ littermates as controls. In the L7-PKCi mouse, the pseudosubstrate $\mathrm{PKC}$ inhibitor, $\mathrm{PKCi}$, is selectively expressed in Purkinje cells under the control of the pcp-2 (L7) gene promoter (De Zeeuw et al., 1998). All animals were bred in a C57BL/6 mouse strain background and were housed in standard conditions ( $12 \mathrm{~h}$ light/ $12 \mathrm{~h}$ dark, water and food ad libitum). Both control and transgenic L7-PKCi mice underwent experiments between 3.5 and 6 months of age. All the behavioral analyses were performed blind with respect to the genotype of the mice. L7-PKCi mice and their control littermates were tested according to the SHIRPA protocol (Crawley, 1999) for comprehensive phenotype assessment prior to the experiment (De Zeeuw et al., 1998; Burguière et al., 2005; van Alphen et al., 2009). All experiments were performed in compliance with European Union Council animal ethics guidelines.

Stimulus-dependent water $Y$-maze conditioning task. Mice were trained in an SWYM apparatus. Each arm, forming a $120^{\circ}$ angle with the adjacent arm, was $50 \mathrm{~cm}$ long and $10 \mathrm{~cm}$ wide, with $30-\mathrm{cm}$-high side walls (Fig. $1 \mathrm{~A}$ ). Arms were filled with water $10 \mathrm{~cm}$ deep. The water was maintained at a temperature of $19^{\circ} \mathrm{C}$ for the duration of the experiment and made opaque by the addition of an inert and nontoxic coloring agent (Acusol OP 301; Brenntag NV). The symmetric configuration of the maze and the high side walls prevented mice from distinguish the arms using either the form of the maze or visual distal cues.

Mice were conditioned to turn right or left depending on the CS. We used two different CS: a light delivered by a white LED $(35,000$ candela intensity) or a sound delivered by a loudspeaker $(5 \mathrm{kHz}, 75 \mathrm{~dB})$. A wrong turn resulted in an air puff on the mouse as an aversive stimulus, which was triggered by the opening of an electromagnetic sluice gate releasing compressed air. This US forced the animal to turn in the correct direction corresponding to the unconditioned response. An escape platform $(8 \mathrm{~cm}$ diameter) was located at the end of the correct arm and hidden $1 \mathrm{~cm}$ below the surface of the water. The US overlapped with the CS according to a classical delay conditioning paradigm. Our paradigm included elements of operant conditioning since the mice learned to turn left according to the sound and right according to the light to avoid the air puff and find the escape platform. The setup was fully automated using an overhead video camera that monitored the mouse's position, determined by a tracking program (SMART, Bioseb). The electronic apparatus controlling the light, the sound, and the air puff was triggered by the position of the animal tracked via an input/output module (supplemental Movie S1, available at www.jneurosci.org as supplemental material).

A day before the start of the training, each animal was put in the water Y-maze apparatus and pretrained with two "forced" right or left configurations in a random order. In these configurations, a barrier blocked the access of one of the two possible arms to force the animal to turn to the open arm. This pretraining day was performed without any stimuli. The aim was to habituate mice to swim in the maze and find the escape platform located at the end of either arm.

The training consisted of two daily sessions of 20 trials each spaced by a minimum of $2 \mathrm{~h}$. The intertrials interval was $30 \mathrm{~s}$ and a whole session lasted a maximum of $15 \mathrm{~min}$. Two configurations were designed with the
SMART program; the right one associated the light with a right turn and the left one associated the sound with a left turn (Fig. $1 B$ ). Each configuration was randomly presented 10 times per session. If the animal made an incorrect turn, an air puff was projected on the mouse at the entrance of the wrong arm. In both configurations, the CS was automatically triggered when the animal crossed the middle of the departure arm and entered the poststimulus zone (Fig. $1 B$ ). Three different zones were distinguished in the departure arm: a starting zone where the animal was released, a prestimulus zone beginning $5 \mathrm{~cm}$ before the middle distance line, and a poststimulus zone ending $24 \mathrm{~cm}$ after the middle distance line.

Because we were particularly interested in the conditional response induced in this protocol, we needed a control experiment mimicking the learning performances but without any conditioning. To do so we trained two other groups of animals (mutants and wild-type) in a yoked task with the same general conditions as their trained partners but without consistent association between stimulus and response (Fig. 1C). For this purpose, we used the performances of conditioned animals to calculate the mean percentage of correct turns for each session of the entire population. With these theoretical values, the mice were exposed to the same amount of light or sound stimulus (randomly presented), air puff, and reward, but without a consistent link between the occurrence of stimulus and the choice of turn. Thus, the mice could not develop an association between CS and the location of the platform at the end of one arm. In the yoked task, a large majority of mice showed a lateralization over the training. The mice that did not develop this behavior tended to turn abnormally in the departure arm, most likely because of the stress of receiving an air puff. We excluded the mice that developed this behavior for consistent analysis of the body rotation ( $<30 \%$ of the mice).

Several parameters were used to characterize the behavior of mice in this new SWYM task. The first parameter was the percentage of correct turns in each session. To assess the mice's abilities to adjust their trajectory during the training, we measured the body rotation in the prestimulus and poststimulus zones. For a given position $(P)$, we calculated the absolute value of the angle between the two vectors $\overrightarrow{(P-1) P}$ and $\overrightarrow{P(P+1)}$. The range of the body rotation, therefore, was from 0 (straightforward) to 180 (U-turn) degrees. We calculated the average body rotation and the body rotation every centimeter in the departure arm.

Data processing and statistical analysis. The sampling of the data was performed every $40 \mathrm{~ms}$ (SMART). Data processing (e.g., the computa- 


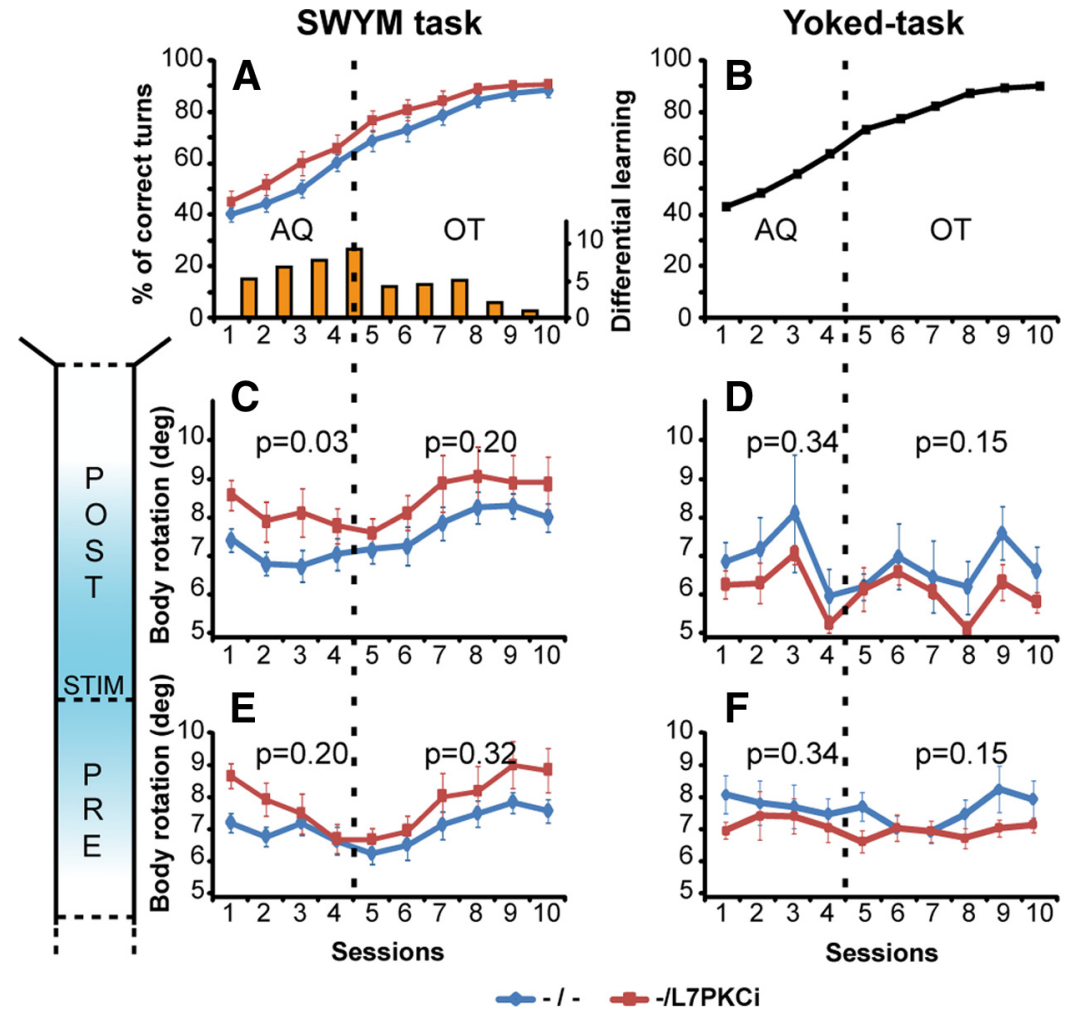

Figure 2. L7-PKCi mice were not impaired in the stimulus-response association. $A$, SWYM conditioning task resulted in quick learning for both groups (ANOVA, $\left.F_{(9,252)}=87.68, p<0.001\right)$. An acquisition phase ( $\mathrm{AQ}$, from sessions $1-4$ ) with a percentage of correct turns $<72.5 \%$ and an overtraining phase (0T, from sessions $5-10$ ) were distinguished (separated by a vertical dotted line). The mean percentage of correct turns in control $(n=9)$ and mutant $(n=12)$ mice were similar during both the acquisition $\left(F_{(1,28)}=2.64, p=0.12\right)$ and the overtraining $\left(F_{(1,28)}=1.61, p=0.22\right)$ phases. A distinction between the acquisition and overtraining phases was also shown here by calculating the difference in the percentage of correct turns across the sessions (right, orange histogram). Improvement from session to session was higher during the first four sessions (acquisition) than the last sessions (overtraining). $\boldsymbol{B}$, Mean value of correct turns of the whole population $(n=21)$ used in the pseudo-yoked task. $\boldsymbol{C}-\boldsymbol{F}$, Mean body rotations observed over the 10 sessions of training for the prestimulus and poststimulus zones in the SWYM and yoked tasks. The mutant mice presented a body rotation significantly higher than control mice during the acquisition phase in the poststimulus zone $\left(F_{(1,28)}=5.56, p=0.026, C\right)$. We did not observe a significant difference between mutants and controls in the prestimulus zone and in the yoked task (D-F). Error bars indicate SEM. STIM, Stimulus.

tion of the assessed parameters) was automated via MATLAB batch programs developed in our laboratory. Statistical studies were performed with the Statview 5.0 software by repeated-measure ANOVA. The significant threshold of tests was fixed at $5 \%(p<0.05$ was significant). All data are presented as mean \pm SEM.

\section{Results}

Inactivation of the cerebellar PF-PC LTD did not impair the stimulus-response association

We evaluated the ability of the L7-PKCi mice to learn the correct stimulus-response association by observing which right or left arm they chose according to the occurring stimulus (Fig. 1). An animal was considered to have learned the stimulus-response association when it reached the criterion of $80 \%$ of correct turns $\left(p<0.05, \chi^{2}\right.$ test) (Jog et al., 1999). After 10 sessions, $93.3 \%$ of all the animals acquired the conditioning (supplemental Movie S1, available at www.jneurosci.org as supplemental material), i.e., L7-PKCi mice (14 of 14) and their control littermates (14 of 16) reached and maintained for at least $3 \mathrm{~d}$ the $80 \%$ criterion $(p>$ $0.05, \chi^{2}$ test). We observed a rapid and significant learning for both groups over the 10 sessions (ANOVA, $F_{(1,252)}=87.68, p<$ 0.001 ) (Fig. 2A). We could distinguish two main phases during animals training: an acquisition phase and an overtraining phase characterized by an inflection point on the learning performance on session 5 (Fig. 2A,B). This is also illustrated by a decrease in the difference in percentage of correct turns between sessions after session 5 (Fig. $2 A$, see histogram).

We compared abilities of the L7-PKCi mice and their control littermates to learn the conditional S-R associations. Both mutant and control groups learned the $\mathrm{S}-\mathrm{R}$ association within the acquisition period $\left(\right.$ ANOVA, $F_{(4,112)}=41.11, p<$ 0.001 ). We did not observe any significant difference between the groups in their ability to acquire the correct CR. Their performances were similar during the acquisition (ANOVA, $F_{(1,28)}=2.64$, $p=0.12)$ and the overtraining phases (ANOVA, $F_{(1,28)}=1.61, p=0.22$ ) (Fig. $2 \mathrm{~A}$ ). To ensure that the level of conditioning was similar for both stimuli, we compared the performance of controls and mutants to distinguish the two CS. Because no significant differences were observed in the acquisition (ANOVA, $F_{(1,48)}=1.5, p=$ 0.22 ) or overtraining (ANOVA, $F_{(1,48)}=$ $1.8, p=0.18$ ), we conclude that mice learned the task similarly with both stimuli.

\section{L7-PKCi transgene expression altered mice trajectories during the acquisition phase}

Although transgenic and control mice showed a similar learning of the stimulus-response association, we questioned their ability to perform an efficient behavioral response. To determine this, we analyzed their trajectory in the prestimulus and poststimulus zone where the conditioned turn was initiated.

To characterize the efficiency of the trajectories, we calculated for each mouse the mean level of body rotations. This parameter indicated the efficiency of the trajectory, in that smaller body rotations correspond to a smoother trajectory with fewer fluctuations. The mutant mice had a higher average level of mean body rotation compared with controls in the poststimulus zone during the acquisition phase (ANOVA, $F_{(1,28)}=5.56, p=0.026$ ) but not during the overtraining phase (ANOVA, $F_{(1,28)}=1.74, p=0.20$ ) (Fig. 2C). This difference was observed during the SWYM conditioning task but not in the control yoked task where no consistent CS-CR association could have been learned during acquisition (ANOVA, $F_{(1,39)}=0.27, p=0.61$ ) or overtraining (ANOVA, $\left.F_{(1,39)}=0.93, p=0.34\right)($ Fig. $2 D)$.

The mean body rotations in the prestimulus zone were similar between mutants and controls in both SWYM (ANOVAs; acquisition, $F_{(1,28)}=1.59, p=0.20$; overtraining, $\left.F_{(1,28)}=1.03, p=0.32\right)$ (Fig. $2 E$ ) and yoked task (ANOVAs; acquisition, $F_{(1,39)}=0.01, p=$ 0.98 ; overtraining, $F_{(1,39)}=0.18, p=0.68$ ) (Fig. $2 F$ ). Again, this result confirmed that the difference observed in the SWYM task was only observed in the poststimulus zone when a conditioned turn was elicited, resulting from the associative-learning procedure.

The mean speed was similar between L7-PKCi and control mice during all the phases (ANOVAs; acquisition, $F_{(1,28)}=0.15$, $p=0.70$; overtraining, $\left.F_{(1,28)}=0.75, p=0.39\right)$. Together, these 
results showed that $\mathrm{L} 7-\mathrm{PKCi}$ mice executed their conditioned turn response less efficiently relative to controls during acquisition of the SWYM task.

The altered trajectories of the L7-PKCi mice were specifically observed during the execution of the conditioned response

Using a centimeter-by-centimeter analysis of the body rotation, we characterized the dynamics of the CR in the poststimulus zone. We first performed this analysis during the acquisition phase (trials 1-80) of the SWYM task (Fig. 3A) and the yoked task (Fig. 3B). In the SWYM task, we observed a clear increase of the body rotation in both groups after the stimulus occurrence (ANOVA, $F_{(22,616)}=46.78, p<$ 0.001 ) (Fig. 3A). The increase of the body rotation in the poststimulus zone was consistent with the initiation of the CR turn triggered by the conditional stimulus. This phenomenon was well illustrated by representing the body rotation in the poststimulus zone relative to the mean body rotation in the prestimulus zone (supplemental Fig. S1 A, available at www. jneurosci.org as supplemental material). Remarkably, we observed a difference between mutants and controls, especially in the poststimulus zone (ANOVA, $F_{(1,28)}=$ $5.51, p=0.026$ ) (Fig. $3 A$ ). In fact, the relative body rotation in the poststimulus zone showed a higher variability in the mutants compared with the controls when they performed their CR (supplemental Fig. S1 $A$, available at www. jneurosci.org as supplemental material). An analysis of the body rotation for each session showed that the difference between mutants and controls in the SWYM task was observed mainly during the first three sessions of the acquisition phase (Fig. 3C,E, G,I). In contrast with the SWYM task, the body rotation tended to be homogeneous in the yoked task, where no conditioned turn could be acquired (Fig. $3 B, D, F, H$, ; supplemental Fig. S1 $B$, available at www.jneurosci.org as supplemental material).

These detailed analysis indicated that L7-PKCi mice had a higher body rotation during the execution of the CR turn, especially at the beginning of the acquisition. Later in the training, the differences between the mutant and wild-type mice tended to disappear. This phenomenon was shown in the centimeter-by-centimeter analysis performed on the overtraining phase of the SWYM task (i.e., average of trials 81-200) (Fig. 4A). Here, the difference observed in the acquisition phase of the SWYM task was not observed in the overtraining phase when animals had acquired and stabilized their CR turns (ANOVA, $\left.F_{(1,28)}=1.16, p=0.28\right)$ (Fig. $4 A$ ). The observed.

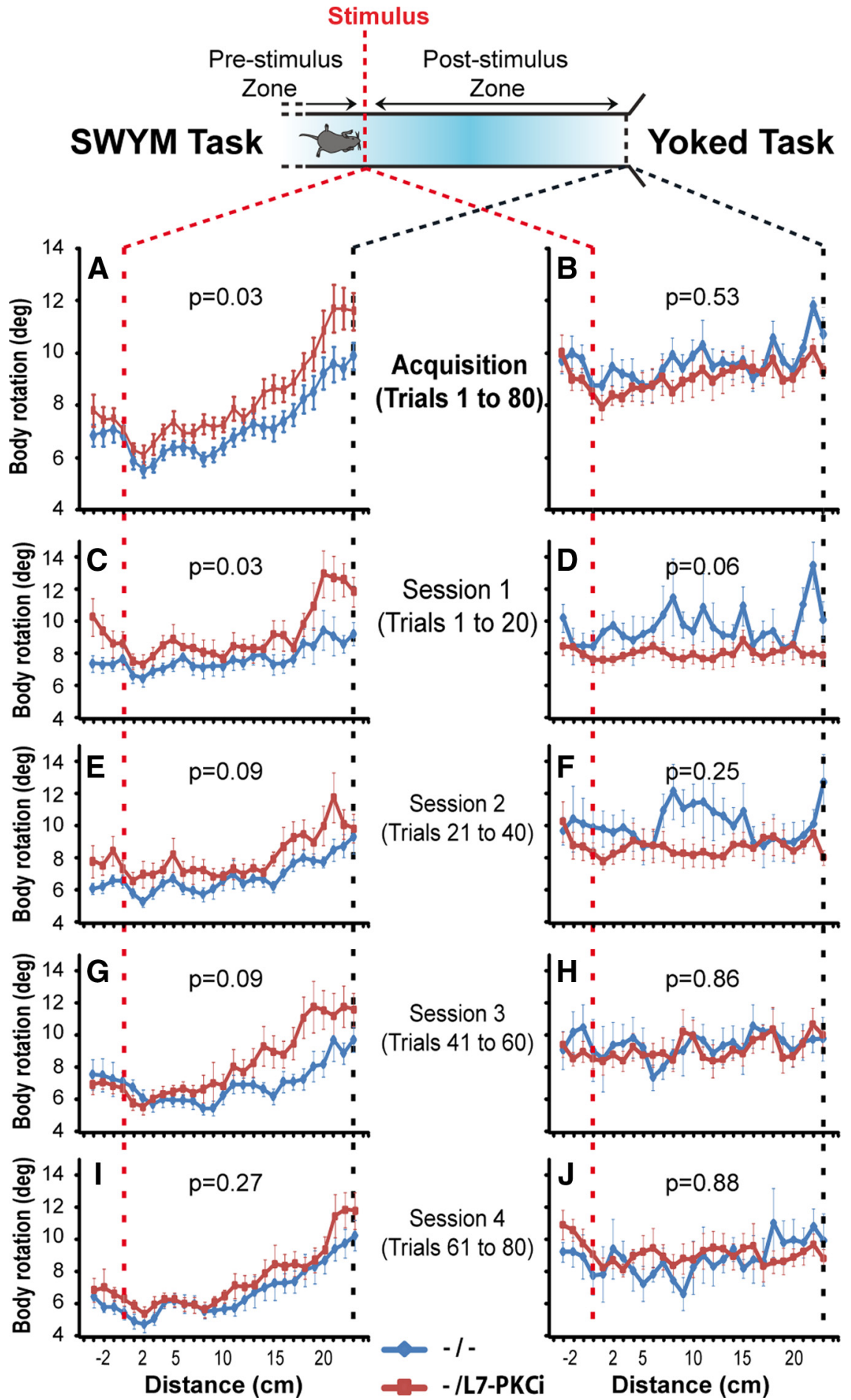

Figure 3. L7-PKCI mice showed suboptimal conditioned responses compared with controls during the acquisition phase. $\boldsymbol{A}, \boldsymbol{B}$, Body rotation profiles of L7-PKCI mice were significantly higher than control mice during the acquisition phase in the SWYM task $\left(F_{(1,28)}=5.2, p=0.026\right)$ but not in the yoked task $\left(F_{(1,15)}=0.4, p=0.53\right) . C, E, G, I$, Session-by-session analyses for the acquisition phase. Differences were observed in sessions $1-3$ for the SWYM condition. $\boldsymbol{D}, \boldsymbol{F}, \boldsymbol{H}, \boldsymbol{J}$, In the yoked task no difference was general aspect of the body rotation in the poststimulus zone relative to the mean body rotation of the prestimulus zone became more identical between mutant and control animals (supplemental Fig. S1C, available at www.jneurosci.org as supplemental material). An analysis for each session showed that no difference was observed in any of them between the two groups (Fig. 4C, E, G, $I, K, M$ ). Consistent with the deficit being 


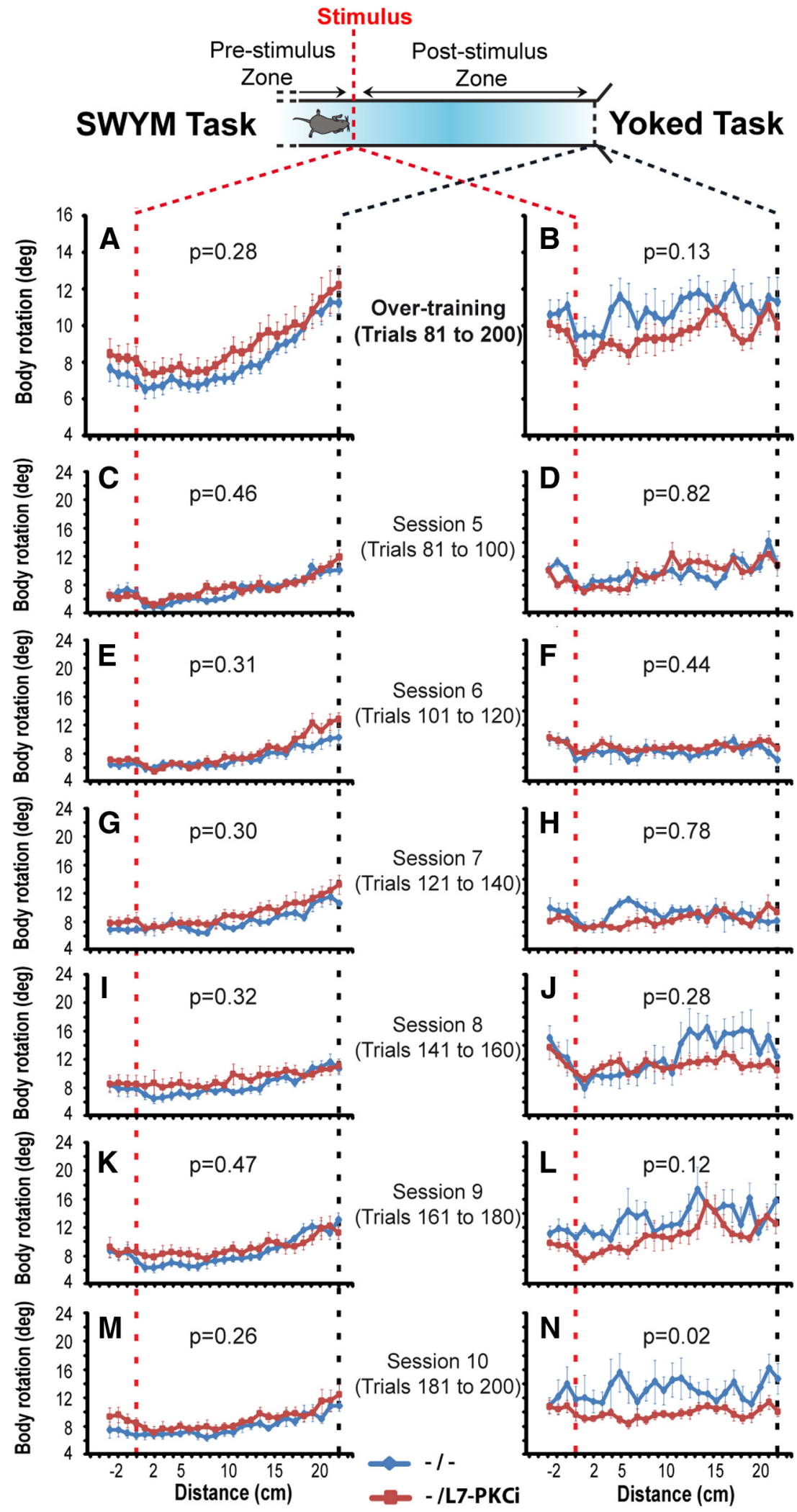

Figure 4. L7-PKCI mice showed no difference compared with controls in the overtraining phase. $A$, Performances of mutant mice were similar to those of control animals during the overtraining phase in the SWYM task $\left(F_{(1,28)}=1.19, p=0.28\right)$. $\boldsymbol{B}$, As expected, no differences were observed in the yoked task $\left(F_{(1,15)}=2.53, p=0.13\right)$. $\mathbf{C}-\mathbf{N}$, Session-by-session analyses for the overtraining phase. No difference was observed in the SWYM or the yoked task. specific to the CR, no significant change was observed in the yoked task during the overtraining phase compared with the acquisition phase (Fig. $4 B, D, F, H$, $J, L, N$; supplemental Fig. S1 $D$, available at www.jneurosci.org as supplemental material).

\section{Discussion}

The PF-PC LTD mechanism has been proposed to contribute to both motor and cognitive learning (Ito, 2001). However, the exact role of PF-PC LTD in classical conditioning has been recently challenged by studies that failed to alter learning after PF-PC LTD disruption (Welsh et al., 2005). Here we tested the possible role of PF-PC LTD in a goal-directed behavioral task in which L7-PKCi mice have to associate and optimize their motor response depending on the conditioned stimulus. Our results demonstrate that cerebellar Purkinje cells' PKC-dependent processes, including PF-PC LTD, are not required for the stimulus-response association process but are essential to adjust the execution of the conditioned motor response.

We developed the SWYM conditioning task where the CS-CR association protocol was similar to the one used during simple motor conditioning protocols (e.g., eyeblink conditioning or vestibuloocular reflex). In contrast to the traditional conditioned task, the $\mathrm{CR}$ required for the SWYM conditioning task involved a complex multijoint movement. Despite this difference, we observed learning curves comparable to those obtained in simpler classical conditioning with two distinct stages: first, an early and fast acquisition phase characterized by a significant improvement in scores reflecting the associative learning; second a later stage characterized by the optimization of the CR (Nudo et al., 1996; Karni et al., 1998; Martin et al., 2000). Our paradigm included elements of operant conditioning since the animal could avoid the US by performing a correct turn. However, extensive comparisons in eyeblink conditioning using either an electrical shock (classical) or an air puff (operant) as the US produced similar conditioned eyeblink reflexes (Koekkoek et al., 2002, $2003,2005)$. In our task, the average time for the animal to execute its CR was homogeneous throughout the training and performed rapidly $(<1200 \mathrm{~ms})$. These values were consistent with CRs observed in classical and operant conditioning protocols (Christian and Thompson, 2003; Buhusi and Meck, 2005). 
In our study, L7-PKCi and control mice showed a similar ability to perform the stimulus-response association required in the SWYM task (Fig. 2). This result indicates that cerebellar PF-PC LTD and other PKC-dependent mechanisms are not essential for the acquisition of the stimulus-response association per se in a conditioning task. Therefore, the participation of the PF-PC LTD supporting learning processes in conditioning tasks should be carefully considered, as already mentioned by previous studies (Raymond and Lisberger, 1997; Welsh et al., 2005). In fact, Welsh and Harvey (1989, 1991) showed that animals still exhibited CRs when normal functions of the interpositus nucleus were restored after reversible lesion. These results emphasized the role of the cerebellum in regulating the sensorimotor processes necessary for the optimal performance of CRs rather than the learning of the CS-CR association per se. It has been proposed that in classical delay conditioning tasks, other structures than the cerebellar cortex could support the stimulus-response association process such, as the cerebellar nuclei (Woodruff-Pak et al., 1985), the striatum (Jog et al., 1999; Blazquez et al., 2002), and/or hippocampus (Takatsuki et al., 2005).

We then tested the possibility that the Purkinje cells PKCdependent processes are involved in the adaptation of the motor response. Even though the L7-PKCi mice could acquire the conditioned turn, our work demonstrated that they were not as efficient as control during the acquisition phase of the SWYM task. The cerebellum has been proposed to be involved in the elaboration of an accurate CR according to cognitive and/or motor inputs received from other brain areas (Doyon et al., 2002; Fanselow and Poulos, 2005; Takatsuki et al., 2005). We quantified the differences observed between L7-PKCi and control mice using detailed body rotation analysis (Fig. 3). We showed that L7PKCi mice's CRs resulted in a higher body rotation than controls' in the acquisition phase. Although the cerebellum has been proposed to serve as a region critical for forming associations between the CS and US, other views have emphasized a specific role of the cerebellar cortex in the performance of the CR rather than learning per se (Jiménez-Díaz et al., 2004; Bellebaum and Daum, 2009). Our results support this idea and point to a role of the Purkinje cells PKC-dependent mechanisms in the adjustment of a CR rather than for its acquisition per se. Several authors have proposed that the cerebellar cortex (Garcia and Mauk, 1998) is a timing machine required to adjust motor response (McCormick and Thompson, 1984; Raymond et al., 1996; Ivry and Spencer, 2004) and that the Purkinje cells are a key neural substrate of this temporal processing (Fiala et al., 1996; Koekkoek et al., 2003). The idea that the cerebellum could be critical for timing in motor, sensory, and cognitive tasks was initially proposed by electrophysiology and behavioral work on the olivocerebellar system. These studies suggest that a possible basic function of the cerebellum could be to produce temporal patterns (Rondi-Reig et al., 1997; Lang et al., 1999, 2006; Jacobson et al., 2008). Finally, we also observe that the difference between mutant and controls faded with overtraining, suggesting that other mechanisms must have compensated for the deficit. These results are consistent with a previous study wherein L7-PKCi mice compensated for a deficit in the adaptation of the vestibulo-ocular reflex when they were exposed to extended training (van Alphen and De Zeeuw, 2002). The authors proposed that other plastic processes in the cerebellum may compensate for the absence of parallel fiber LTD in the Purkinje cells of L7-PKCi mice when the animals are exposed to long training periods (Lev-Ram et al., 2003; De Zeeuw and Yeo, 2005; Faulstich et al., 2006).

To better characterize the functional role of the cerebellar cortex in such conditioning, it is important to investigate further the role of different PKC-dependent mechanism in future studies. In several mutant models, including the L7-PKCi mouse, the kinases or related proteins affected are also involved in a different process than the induction of LTD. In the L7-PKCi mouse, the Purkinje cell PKC has many substrates that may affect baseline function of the cerebellar network, morphology of the Purkinje cells, and other forms of synaptic and nonsynaptic plasticity in the cerebellum (Kano et al., 1996; Hansel et al., 2006). For example, Purkinje cell dendritic development also critically depends on the activity of PKC. PKC signaling pathways have been shown to be closely linked to the regulation of dendritic growth and its arborization during cerebellar development at different time points (Metzger and Kapfhammer, 2003). $\mathrm{Ca}^{2+}$-dependent PKC subtypes, activated by synaptic inputs through metabotropic glutamate receptors, trigger functional changes as well as long-term anatomical maturation of the Purkinje cell dendritic tree. Many signals, including receptor phosphorylation, gene transcription, Notch and Rho family GTPase signaling, and cytoskeletal rearrangements, modulate the growth of dendrites and are necessary for morphological changes (Scott and Luo, 2001). Moreover, many of these signals are modulated by synaptic activity, supporting the hypothesis that the structure of dendrites can be rapidly modified by synaptic plasticity. Inhibition of PKC could also affect the efficacy of GABA receptors at interneuron-Purkinje cell synapses by influencing their surface density and sensitivity to positive allosteric modulators and/or by modifying chloride conductance (Song and Messing, 2005). Therefore, we can not rule out that the deficit of CR optimization observed in the L7-PKCi mouse model could be due to an impaired PKC-dependent mechanism other than PF-PC LTD. In further studies, the specific role of PF-PC LTD will need to be distinguished from other PKC-dependent mechanisms during the associative-learning process. New mice models may help to answer this issue by targeting the expression of the PF-PC LTD rather than its induction (Steinberg et al., 2006).

In conclusion, we previously proposed that cerebellar PF-PC LTD was necessary for the acquisition of efficient trajectories toward a spatial goal (Burguière et al., 2005; Rondi-Reig and Burguière, 2005). Other studies more computational suggested that the cerebellar cortex could have a crucial role in spatial reorientation and proposed that the simple and complex spike activity, a process potentially critical for long-term adaptation, could be fundamental for heading perception and spatial navigation (Yakusheva et al., 2007; Angelaki et al., 2009). Here, with the SWYM task, we questioned the role of Purkinje cells PKC-dependent processes, such as PF-PC LTD, in the stimulus-response association process and the optimization of the CR in a spatial navigation task. In line with a previous study (Welsh et al., 2005), we conclude that PF-PC LTD is not necessary for the stimulus-response association per se. However, PF-PC LTD and/or other Purkinje cells' PKC-dependent mechanisms were required for the refinement of the motor response. Moreover, we bring additional evidence supporting the idea that the cerebellar cortex could underlie a basic and common process of conditioned motor response optimization required in multijoint motor conditioning tasks (e.g., whole-body rotation response in spatial navigation) as well as in simple classical conditioning tasks (e.g., eyeblink reflex response).

\section{References}

Aiba A, Kano M, Chen C, Stanton ME, Fox GD, Herrup K, Zwingman TA, Tonegawa S (1994) Deficient cerebellar long-term depression and impaired motor learning in mGluR1 mutant mice. Cell 79:377-388.

Angelaki DE, Yakusheva TA, Green AM, Dickman JD, Blazquez PM (2010) Computation of egomotion in the macaque cerebellar vermis. Cerebellum 9:174-182. 
Bellebaum C, Daum I (2009) Mechanisms of cerebellar involvement in associative learning. Cortex. Advance online publication. Retrieved July 1, 2010. doi:10.1016/j.cortex.2009.07.016.

Blazquez PM, Fujii N, Kojima J, Graybiel AM (2002) A network representation of response probability in the striatum. Neuron 33:973-982.

Buhusi CV, Meck WH (2005) What makes us tick? Functional and neural mechanisms of interval timing. Nat Rev Neurosci 6:755-765.

Burguière E, Arleo A, Hojjati M, Elgersma Y, De Zeeuw CI, Berthoz A, RondiReig L (2005) Spatial navigation impairment in mice lacking cerebellar LTD: a motor adaptation deficit? Nat Neurosci 8:1292-1294.

Christian KM, Thompson RF (2003) Neural substrates of eyeblink conditioning: acquisition and retention. Learn Mem 10:427-455.

Crawley JN (1999) The role of galanin in feeding behavior. Neuropeptides 33:369-375.

De Zeeuw CI, Yeo CH (2005) Time and tide in cerebellar memory formation. Curr Opin Neurobiol 15:667-674.

De Zeeuw CI, Hansel C, Bian F, Koekkoek SK, van Alphen AM, Linden DJ, Oberdick J (1998) Expression of a protein kinase C inhibitor in Purkinje cells blocks cerebellar LTD and adaptation of the vestibulo-ocular reflex. Neuron 20:495-508.

Doyon J, Song AW, Karni A, Lalonde F, Adams MM, Ungerleider LG (2002) Experience-dependent changes in cerebellar contributions to motor sequence learning. Proc Natl Acad Sci U S A 99:1017-1022.

Fanselow MS, Poulos AM (2005) The neuroscience of mammalian associative learning. Annu Rev Psychol 56:207-234.

Faulstich M, van Alphen AM, Luo C, du Lac S, De Zeeuw CI (2006) Oculomotor plasticity during vestibular compensation does not depend on cerebellar LTD. J Neurophysiol 96:1187-1195.

Feil R, Hartmann J, Luo C, Wolfsgruber W, Schilling K, Feil S, Barski JJ, Meyer M, Konnerth A, De Zeeuw CI, Hofmann F (2003) Impairment of LTD and cerebellar learning by Purkinje cell-specific ablation of cGMPdependent protein kinase I. J Cell Biol 163:295-302.

Fiala JC, Grossberg S, Bullock D (1996) Metabotropic glutamate receptor activation in cerebellar Purkinje cells as substrate for adaptive timing of the classically conditioned eye-blink response. J Neurosci 16:3760-3774.

Garcia KS, Mauk MD (1998) Pharmacological analysis of cerebellar contributions to the timing and expression of conditioned eyelid responses. Neuropharmacology 37:471-480.

Hansel C, de Jeu M, Belmeguenai A, Houtman SH, Buitendijk GH, Andreev D, De Zeeuw CI, Elgersma Y (2006) alphaCaMKII Is essential for cerebellar LTD and motor learning. Neuron 51:835-843.

Ito M (2001) Cerebellar long-term depression: characterization, signal transduction, and functional roles. Physiol Rev 81:1143-1195.

Ito M, Kano M (1982) Long-lasting depression of parallel fiber-Purkinje cell transmission induced by conjunctive stimulation of parallel fibers and climbing fibers in the cerebellar cortex. Neurosci Lett 33:253-258.

Ivry RB, Spencer RM (2004) The neural representation of time. Curr Opin Neurobiol 14:225-232.

Jacobson GA, Rokni D, Yarom Y (2008) A model of the olivo-cerebellar system as a temporal pattern generator. Trends Neurosci 31:617-625.

Jiménez-Díaz L, Navarro-López Jde D, Gruart A, Delgado-García JM (2004) Role of cerebellar interpositus nucleus in the genesis and control of reflex and conditioned eyelid responses. J Neurosci 24:9138-9145.

Jog MS, Kubota Y, Connolly CI, Hillegaart V, Graybiel AM (1999) Building neural representations of habits. Science 286:1745-1749.

Kano M, Kano M, Fukunaga K, Konnerth A (1996) Ca(2+)-induced rebound potentiation of gamma-aminobutyric acid-mediated currents requires activation of $\mathrm{Ca} 2+/$ calmodulin-dependent kinase II. Proc Natl Acad Sci U S A 93:13351-13356.

Karni A, Meyer G, Rey-Hipolito C, Jezzard P, Adams MM, Turner R, Ungerleider LG (1998) The acquisition of skilled motor performance: fast and slow experience-driven changes in primary motor cortex. Proc Natl Acad Sci U S A 95:861-868.

Koekkoek SK, Den Ouden WL, Perry G, Highstein SM, De Zeeuw CI (2002) Monitoring kinetic and frequency-domain properties of eyelid responses in mice with magnetic distance measurement technique. J Neurophysiol $88: 2124-2133$.

Koekkoek SK, Hulscher HC, Dortland BR, Hensbroek RA, Elgersma Y, Ruigrok TJ, De Zeeuw CI (2003) Cerebellar LTD and learning-dependent timing of conditioned eyelid responses. Science 301:1736-1739.

Koekkoek SK, Yamaguchi K, Milojkovic BA, Dortland BR, Ruigrok TJ, Maex R, De Graaf W, Smit AE, VanderWerf F, Bakker CE, Willemsen R, Ikeda
T, Kakizawa S, Onodera K, Nelson DL, Mientjes E, Joosten M, De Schutter E, Oostra BA, Ito M, De Zeeuw CI (2005) Deletion of FMR1 in Purkinje cells enhances parallel fiber LTD, enlarges spines, and attenuates cerebellar eyelid conditioning in Fragile X syndrome. Neuron 47:339-352.

Lang EJ, Sugihara I, Welsh JP, Llinás R (1999) Patterns of spontaneous purkinje cell complex spike activity in the awake rat. J Neurosci 19:2728-2739.

Lang EJ, Sugihara I, Llinás R (2006) Olivocerebellar modulation of motor cortex ability to generate vibrissal movements in rat. J Physiol 571:101-120.

Lev-Ram V, Mehta SB, Kleinfeld D, Tsien RY (2003) Reversing cerebellar long-term depression. Proc Natl Acad Sci U S A 100:15989-15993.

Linden DJ, Connor JA (1991) Participation of postsynaptic PKC in cerebellar long-term depression in culture. Science 254:1656-1659.

Martin SJ, Grimwood PD, Morris RG (2000) Synaptic plasticity and memory: an evaluation of the hypothesis. Annu Rev Neurosci 23:649-711.

McCormick DA, Thompson RF (1984) Neuronal responses of the rabbit cerebellum during acquisition and performance of a classically conditioned nictitating membrane-eyelid response. J Neurosci 4:2811-2822.

Metzger F, Kapfhammer JP (2003) Protein kinase C: its role in activitydependent Purkinje cell dendritic development and plasticity. Cerebel$\operatorname{lum} 2: 206-214$

Nudo RJ, Milliken GW, Jenkins WM, Merzenich MM (1996) Usedependent alterations of movement representations in primary motor cortex of adult squirrel monkeys. J Neurosci 16:785-807.

Porras-García E, Cendelin J, Domínguez-del-Toro E, Vozeh F, DelgadoGarcía JM (2005) Purkinje cell loss affects differentially the execution, acquisition and prepulse inhibition of skeletal and facial motor responses in Lurcher mice. Eur J Neurosci 21:979-988.

Raymond JL, Lisberger SG (1997) Multiple subclasses of Purkinje cells in the primate floccular complex provide similar signals to guide learning in the vestibulo-ocular reflex. Learn Mem 3:503-518.

Raymond JL, Lisberger SG, Mauk MD (1996) The cerebellum: a neuronal learning machine? Science 272:1126-1131.

Rondi-Reig L, Burguière E (2005) Is the cerebellum ready for navigation? Prog Brain Res 148:199-212.

Rondi-Reig L, Delhaye-Bouchaud N, Mariani J, Caston J (1997) Role of the inferior olivary complex in motor skills and motor learning in the adult rat. Neuroscience 77:955-963.

Scott EK, Luo L (2001) How do dendrites take their shape? Nat Neurosci 4:359-365

Song M, Messing RO (2005) Protein kinase C regulation of GABAA receptors. Cell Mol Life Sci 62:119-127.

Steinberg JP, Takamiya K, Shen Y, Xia J, Rubio ME, Yu S, Jin W, Thomas GM, Linden DJ, Huganir RL (2006) Targeted in vivo mutations of the AMPA receptor subunit GluR2 and its interacting protein PICK1 eliminate cerebellar long-term depression. Neuron 49:845-860.

Takatsuki K, Kawahara S, Mishina M, Kirino Y (2005) Characterization of hippocampal theta rhythm in wild-type mice and glutamate receptor subunit delta 2 mutant mice during eyeblink conditioning with a short trace interval. Brain Res 1063:159-167.

Thompson RF, Steinmetz JE (2009) The role of the cerebellum in classical conditioning of discrete behavioral responses. Neuroscience 162:732-755.

van Alphen AM, De Zeeuw CI (2002) Cerebellar LTD facilitates but is not essential for long-term adaptation of the vestibulo-ocular reflex. Eur J Neurosci 16:486-490.

van Alphen B, Winkelman BH, Frens MA (2009) Age- and sex-related differences in contrast sensitivity in C57BL/6 mice. Invest Ophthalmol Vis Sci 50:2451-2458.

Welsh JP, Harvey JA (1989) Cerebellar lesions and the nictitating membrane reflex: performance deficits of the conditioned and unconditioned response. J Neurosci 9:299-311.

Welsh JP, Harvey JA (1991) Pavlovian conditioning in the rabbit during inactivation of the interpositus nucleus. J Physiol 444:459-480.

Welsh JP, Yamaguchi H, Zeng XH, Kojo M, Nakada Y, Takagi A, Sugimori M, Llinás RR (2005) Normal motor learning during pharmacological prevention of Purkinje cell long-term depression. Proc Natl Acad Sci U S A 102:17166-17171.

Woodruff-Pak DS, Lavond DG, Thompson RF (1985) Trace conditioning: abolished by cerebellar nuclear lesions but not lateral cerebellar cortex aspirations. Brain Res 348:249-260.

Yakusheva TA, Shaikh AG, Green AM, Blazquez PM, Dickman JD, Angelaki DE (2007) Purkinje cells in posterior cerebellar vermis encode motion in an inertial reference frame. Neuron 54:973-985. 